\section{Neue Buchreihe}

\section{MANFRED L. PiRner}

Im Zusammenhang mit der Veröffentlichung der Tagungsdokumentation zur IZMM-Tagung 2002, "Homo medialis. Perspektiven und Probleme einer Anthropologie der Medien", wurde eine neue Buchreihe mit dem Titel "medienpädagogik intendisziplinär" ins Leben gerufen. Herausgeber sind die an der Pädagogischen Hochschule Ludwigsburg tätigen Wissenschaftler Horst Niesyto (Professor für Erziehungswissenschaft mit Schwerpunkt Medienpädagogik), Manfred L. Pirner (Professor für ev. Theologie und Religionspädagogik) und Matthias Rath (Professor für Philosophie).

A us dem Reihen-H erausgeber- $V$ orwort zum Buch "H omo medialis" :

Angesichts der Komplexität des Mediensystems in modernen $\mathrm{G}$ esellschaften und angesichts der Bedeutung, welche die Medien für nahezu alle Bereiche unseres Lebens gewonnen haben, sind Medienforschung und Medienpädagogik vorzugsweise interdisziplinär zu betreiben. In Bezug auf die Praxis der Medienerziehung und Medienbildung lassen sich inzwischen viele Reihen und Zeitschriften finden, die eine Verständigung über das gemeinsame Praxisfeld ermöglichen. Ein Disziplinen übergreifender Forschungszusammenhang jedoch verlangt verstärkt nach theoriebildender Arbeit, die erst noch im Wachsen ist. Diese Einsicht führte im Jahr 1998 zur Gründung des "Interdisziplinären Zentrums für Medienpädagogik und Medienforschung" (IZMM) an der Pädagogischen Hochschule Ludwigsburg. A uf Initiative von Horst Niesyto wurde so ein Diskussions- und Arbeitszusammenhang geschaffen, um die Kooperation zwischen interessierten Wissenschaftlerinnen und Wissenschaftlem im Bereich Medienpädagogik und Medienforschung instituts- und fakultätsübergreifend zu intensivieren.

Neben regelmäßigen Arbeitstreffen, Lehrveranstaltungsreihen, Forschungskolloquien und Gastvorträgen wurden in den vergangenen Jahren mehrere Fachtagungen durchgeführt. Ergebnisse aus diesen Forschungs- und Diskussionszusammenhängen sind teilweise bereits im kopaed-Verlag erschienen ("Selbstausdruck mit Medien. Eigenproduktion mit Medien als Gegenstand der Kindheits- und Jugendforschung", "Videoculture. Video und interkulturelle Kommunikation", beide hg. von Horst Niesyto). Außerdem wird seit zwei Jahren eine OnlineZeitschrift, die "Ludwigsburger Beiträge zur Medienpädagogik" herausgegeben, die halbjährlich erscheint (www.ph-ludwigsburg.de/ medien1/ intzent.htm).
Mit der neuen Buchreihe "medienpädagogik interdisziplinär", deren erster Band hier vorliegt, soll ein Forum geboten werden, das über die Tagungsdokumentationen und sonstigen Veröffentlichungen des IZMM hinaus auch anderen medienpädagogischen Arbeiten mit interdisziplinären Akzenten aus dem ganzen deutschen Sprachraum offen steht. Wir hoffen, dass die Vernetzung der medienpädagogischen Forschungen und Theoriebildungen dadurch weiter vorangetrieben und Medienbildung in möglichst vielfältigen schulischen und außerschulischen Kontexten verankert werden kann.

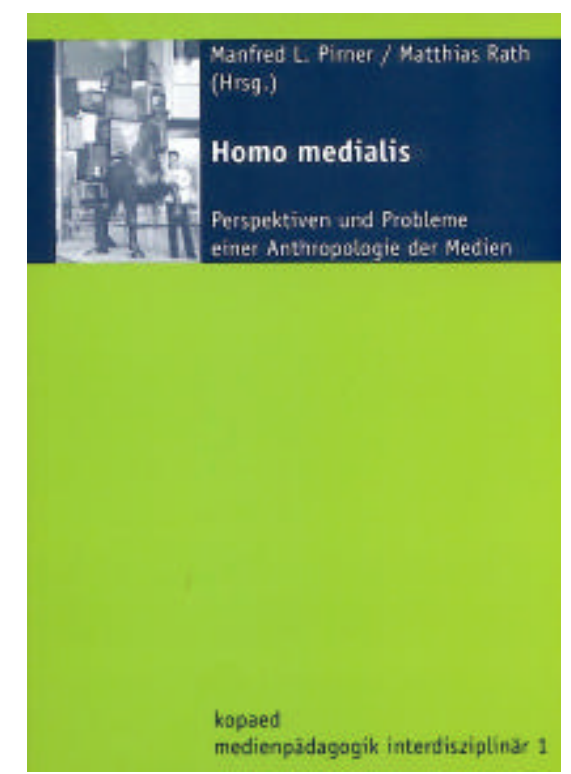

